\title{
Role of chemotherapy in the management of locally advanced carcinoma cervix
}

\author{
Huma Firdaus ${ }^{1}$, Ayanat Husain ${ }^{2 *}$
}

\begin{abstract}
${ }^{1}$ Department of Obstetrics and Gynecology, ERA's Lucknow Medical College and Hospital, Lucknow, Uttar Pradesh, India

${ }^{2}$ Department of Surgery, Integral Institute of Medical Sciences and Research, Lucknow, Uttar Pradesh, India
\end{abstract}

Received: 02 June 2018

Accepted: 28 June 2018

\author{
*Correspondence: \\ Dr. Ayanat Rizvi, \\ E-mail: ayanatrizvi@gmail.com
}

Copyright: () the author(s), publisher and licensee Medip Academy. This is an open-access article distributed under the terms of the Creative Commons Attribution Non-Commercial License, which permits unrestricted non-commercial use, distribution, and reproduction in any medium, provided the original work is properly cited.

\begin{abstract}
Background: This study was undertaken to evaluate role of chemotherapy in the management of locally advanced carcinoma cervix in J.N. Medical College Hospital, A.M.U. Aligarh.

Methods: A total of 50 cases were studied. 25 cases received radiotherapy alone or surgery followed by radiotherapy. In remaining 25 cases, 18 patients received neoadjuvant chemotherapy followed by surgery or radiotherapy where as seven patients out of 25 received concurrent chemo-radiation. SPSS software was used for data analysis.

Results: Mean age of patient was 51.5 years. Majority of patients $(60 \%)$ were in FIGO clinical stage IIB followed by stage IIIB (20\%), stage IVA (12\%) and stage IIA (8\%). Out of 25 patients receiving chemotherapy, 68\% showed CR whereas $20 \%$ showed PR, $4 \%$ showed minimal response and $8 \%$ showed PD while on chemotherapy. In radiotherapy group, CR was found in $56 \%$ and PR in $36 \%$ of cases.

$8 \%$ of patients showed PD while on radiotherapy. Patients with stage IIB disease showed best response with CR in $70 \%$ and PR in $16.66 \%$ cases. $6.66 \%$ of patients showed SD and $6.66 \%$ showed PD while on therapy. $55.55 \%$ of patients with SCC showed CR and $33.33 \%$ showed PR. In adenocarcinoma, CR was seen in $100 \%$ cases. In adenosquamous carcinoma, $66.66 \%$ showed CR whereas $33.33 \%$ showed PR. $61.9 \%$ of well differentiated carcinoma showed CR and $28.57 \%$ showed PR. $4.76 \%$ showed SD and $4.76 \%$ showed PD. $58.33 \%$ of moderately differentiated carcinoma showed CR, $37.5 \%$ showed PR and $4.17 \%$ showed SD. $20 \%$ of poorly differentiated carcinoma showed CR, $60 \%$ showed PR and PD was seen in $20 \%$ cases. Patients with bulkly tumours in present study, irrespective of their response to chemotherapy, appeared to have worse prognosis than patients with more advanced pelvic disease. These findings suggest that the biology of bulky stage lb, IIa \& IIb disease may be more aggressive than that of more extensive locally advanced disease.

Conclusions: Chemotherapy can be considered as an adjuvant to available modalities of treatment, like surgery or radiotherapy, to improve the overall survival of cancer cervix patients.
\end{abstract}

Keywords: Cancer cervix, Cervix, Chemotherapy

\section{INTRODUCTION}

Cervical cancer is the second most common cancer in Indian women aged 15-44 years. Every year in India, 122,844 women are diagnosed with cervical cancer and 67,477 dies from the disease. ${ }^{1}$ Various modalities of treatment are available for cancer cervix. The two modalities for primary treatment are surgery and radiotherapy. Whereas radiation therapy can be used in all stages of disease, surgery alone is limited to patients with stages I and 11 a disease. In the past chemotherapy was used only for palliative purpose in advanced disease. Based on the type of response obtained in initial trials of chemotherapy in cases of carcinoma cervix, it started 
appearing logical to accept chemotherapy to be an effective form of therapeutic intervention in the management of cervical cervix. In the last few years, neoadjuvant chemotherapy has been applied as a new therapeutic approached in locally advanced cervical cancers because of disappointing results with conventional treatments. Clinical trials using neoadjuvant chemotherapy followed by surgery reported that the reduction of tumour bulk could make radical surgery possible in a high percentage of cases previously considered inoperable. ${ }^{2}$ Neoadjuvant chemotherapy given before definitive surgery also increases the potential for local control because the cells shed at the time of surgery would be less viable for implantation, which is the major cause of recurrence. ${ }^{3}$ The US National Cancer institute strongly recommends the use of chemotherapy in cases of cancer cervix as the first line of treatment followed by surgery or radiotherapy. This reduces the risk of death by 30 to $50 \%$ as compared to conventional therapy. ${ }^{4}$ Concurrent or concomitant chemotherapy is given during radiotherapy to increase the response rate to radiation. The National Cancer institute has recommended on the basis of clinical data based on results available worldwide, that it should be used in all cases of locally advanced or bulky tumours. ${ }^{5}$ With this background this study was undertaken to find out the incidence of early, advanced cancer cervix and recurrence of cancer cervix of carcinoma cervix at J.N. Medical College Hospital and to evaluate the role of chemotherapy in locally advanced cancer cervix.

\section{METHODS}

The study was carried out in the department of obstetrics and Gynaecology in association with the Department of Pathology and

Radiotherapy in J.N. Medical collage Hospital, AMU Aligarh.

Patients with histologically confirmed carcinoma cervix Stage IB to IVA having Haemogram, renal function test, liver function test and coagulation profile within normal range were included.

Patients who were included were divided into two groups.

\section{Study group}

Subdivided into three groups depending upon the type of chemotherapy received.

- Neoadjuvant chemotherapy.

- Concurrent chemotherapy.

- Surgery + concurrent chemotherapy

\section{Control group}

Subdivided into two groups
- Radiotherapy alone

- Surgery + postoperative radiotherapy

After taking detailed history, general and systemic examination was done. Gynaecological examination including perabdomen, pervaginal and perectal examination was done. Patients were then staged using the clinical staging system proposed by the International federation of gynaecology and obstetrics (F1GO) and patients with locally advanced inoperable tumours (Stages IB-IVA) were eligible for first line treatment with chemotherapy.

Following chemotherapeutic protocols were given in consultation with medical oncologists depending upon the clinical stage, general wellbeing of patient and available financial recourse.

\section{Neoadjuvant chemotherapy regime}

- Injection Cisplatin $75 \mathrm{mg} / \mathrm{m} 2$ BSA in divided doses on Day 1 and 2

- Injection Methotrexate $-25 \mathrm{mg} / \mathrm{m} 2$ BSA on Day 1

Two such cycles were given at an interval of three weeks. Response of regime was studied after two weeks of each course of chemotherapy before next course was due and after completion of chemotherapy and complete definitive therapy including radiotherapy or surgery, by doing clinical examination and relevant investigations.

\section{Concurrent Chemotherapy}

\section{Protocol 1}

Cisplatin only regime (Table): Cisplatin $35 \mathrm{mg} / \mathrm{m} 2 \mathrm{BSA}$ given weekly along with five fractions of radiation in a week. For 5-6 cycles.

\section{Protocol 2}

Cisplatin 5-fluorouracil regime (Table:): Cispatin (50mg) IVI infusion + 5 Fluorouracil (750mg) IVI infusion

Five to six cycles of chemotherapy were given weekly along with 5 fractions of radiation in a week

The assessment of tumour response was done according to WHO criteria of tumour response:

- $\quad$ Subjective response: Includes symptomatic relief e.g. relief of pelvic pain, discharge or bleeding per vaginum and improvement in general Condition, etc.

- Objective response: It is graded as follows (WHO criteria)

1. Complete response (CR): It is defined as disappearance of all measurable disease.

2. Partial response (PR): It is defined as a decrease in tumour size equal to or greater then $50 \%$ of 
the product of the two maximal tumour diameters.

3. Stable disease (SD): is defined as less than $50 \%$ regression of of tumour.

4. Progressive disease (PD): It is defined as a greater than $25 \%$

Increase in size of tumour or appearance of new lesions. Moreover, acceptability and toxicity of given regimen was studied in each patient. After completing chemotherapy, majority of the patients were treated with pelvic radiotherapy but in some patients with a dramatic reduction in tumour size, surgery was performed, and radiotherapy was administered subsequently to those with lymphnode metastasis.

Pelvic radiography: Whole pelvic radiation 45-50 Gy in 4.5-5 weeks was given subsequent chemotherapy. In patients with disease progression chemotherapy was stopped and radiotherapy commenced immediately.

\section{RESULTS}

Out of a total of 830 patients admitted, 84 had been clinically diagnosed as cancer cervix and 33 were doubtful.

The incidence of cervical cancer in present study was $16.96 \%$. Total number of cervical biopsies sent in a same year was 116. Out of them, $62(53 \%)$ cervical biopsies were positive fro malignancy. Maximum number of patients $18(36 \%)$ were in the age group 41-50. Mean age of patient was 51.5 years.
Table 1: Geographical distribution of patients.

\begin{tabular}{|lll|}
\hline Residential Area & No. of patients & $\%$ \\
\hline Urban & 21 & 42 \\
\hline Rural & 29 & 58 \\
\hline Religion & & \\
\hline Hindu & 36 & 74 \\
\hline Muslim & 13 & 26 \\
\hline Others & 1 & 2 \\
\hline Class & & \\
\hline Upper & 0 & 0 \\
\hline Upper middle & 1 & 2 \\
\hline Lower middle & 19 & 38 \\
\hline Lower & 30 & 60 \\
\hline Age of marriage & & \\
\hline$\leq 18$ years & 30 & 60 \\
\hline $19-25$ years & 19 & 38 \\
\hline$>25$ & 1 & 2 \\
\hline
\end{tabular}

Maximum number of patients $30(60 \%)$ were in FIGO stage IIB followed by stage IIIB with $10(20 \%)$ patients. $6(12 \%)$ patients had stage IVA disease followed by 4 $(8 \%)$ patients in stage IIA. Higher percentage of patients (58\%) were from rural areas and $42 \%$ belong to urban areas. Higher incidence (74\%) of carcinoma cervix was found in Hindu population. Maximum number of patients $(60 \%)$ were of lower socio-economic status, $38 \%$ in lower middle class and only $2 \%$ in upper middle class. Maximum number of patients $(60 \%)$ got married at the age of 18 years or less. This shows that risk of carcinoma cervix is inversely related to age at first intercourse. (Table 1).

Table 2: Objective response (WHO criteria) $\mathbf{n}=\mathbf{5 0}$.

\begin{tabular}{|c|c|c|c|c|c|c|c|c|c|}
\hline Groups & No. of points & CR & CR\% & PR & PR \% & SD & SD \% & PD & PD \% \\
\hline \multicolumn{10}{|l|}{ Study group } \\
\hline Neoadjuvant CT + RT & 16 & 10 & 62.5 & 4 & 25 & 0 & 0 & 2 & 12.5 \\
\hline Neoadjuvant CT + surgery & 2 & 2 & 100 & 0 & 0 & 0 & 0 & 0 & 0 \\
\hline Concurrent CR & 7 & 5 & 71.4 & 1 & 14.3 & 1 & 14.3 & 0 & 0 \\
\hline \multicolumn{10}{|l|}{ Control group } \\
\hline RT alone & 19 & 10 & 52.62 & 17 & 36.84 & 2 & 10.53 & 0 & 0 \\
\hline Surgery $+\mathrm{RT}$ & 6 & 4 & 66.66 & 2 & 33.33 & 0 & 0 & 0 & 0 \\
\hline
\end{tabular}

CR: Complete response, SD: Stable disease, PR: Partial response, PD: Progressive disease

Out of 25 patients receiving chemotherapy. 68\% showed CR whereas $20 \%$ showed PR. $4 \%$ showed minimal response whereas $8 \%$ showed $\mathrm{PD}$ while on chemotherapy. In radiotherapy group, CR was found in $58 \%$ and PR was found in $36 \%$ of patients. $8 \%$ showed SD while on radiotherapy. The pattern of recurrence is shown in above table. Out of 25 patients, $9(36 \%)$ patients relapsed. Recurrence was suspected in the pap smears on follow up by the presence of small dark tumor cells and large naked nuclei. There was a marked difference in relapse rates between chemotherapy responders and non-responders. Both the patients who were non-responders to chemotherapy have relapsed $(100 \%)$, one with local recurrence and one with both local and distant metastasis. Out of 23 chemotherapy responders, $30.4 \%$ have relapsed, one with distant metastasis and five with local recurrence only. Patients in stage IIB disease showed best response with CR in $70 \%$ and PR in $16.66 \%$. 6.66\% showed SD and $6.66 \%$ showed $\mathrm{PD}$ while on therapy. CR was seen in maximum number of patients with Stage IIB disease followed by stage IIA and stage IIIB and markedly less with stage IVA disease. 
Table 3: Response to chemotherapy and subsequent outcome $n=25$.

\begin{tabular}{|c|c|c|c|c|c|c|}
\hline \multirow{2}{*}{$\begin{array}{l}\text { Response to NACT/Concurrent } \\
\text { chemoradiation }\end{array}$} & \multirow[t]{2}{*}{ Total } & \multirow[t]{2}{*}{ Surgery } & \multirow[t]{2}{*}{ Surgery+ adjuvant RT } & \multirow[t]{2}{*}{$\mathbf{R T}$} & \multicolumn{2}{|c|}{ Recurrence } \\
\hline & & & & & Local & Distant \\
\hline Complete response & 8 & 2 & 1 & 6 & 1 & 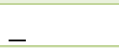 \\
\hline Partial response & 15 & _ & _- & 15 & 5 & $\overline{1}$ \\
\hline Stable disease & 1 & & - & 1 & 1 & 1 \\
\hline Progressive disease & 1 & _- & - & 1 & 1 & - \\
\hline
\end{tabular}

Table 4: Response of CT+RT according to clinical stage $(n=50)$.

\begin{tabular}{|llllllllll|}
\hline Figo stage & No. of patients & CR & CR \% & PR & PR \% & SD & SD \% & PD & PD \% \\
\hline IIA & 4 & 2 & 50 & 2 & 50 & 0 & 0 & 0 & 0 \\
\hline IIB & 30 & 21 & 70 & 5 & 16.66 & 2 & 6.66 & 2 & 6.66 \\
\hline IIIA & 0 & 0 & 0 & 0 & 0 & 0 & 0 & 0 & 0 \\
\hline IIIB & 10 & 9 & 40 & 5 & 50 & 1 & 10 & 0 & 0 \\
\hline IVA & 6 & 2 & 33.33 & 4 & 66.66 & 0 & 0 & 0 & 0 \\
\hline
\end{tabular}

\section{DISCUSSION}

Maximum number of patients, $18(36 \%)$ were in the age group 41-50 years. The peak age of occurrence of cervical cancer in India is between 55 and 59 years, and the highest age-adjusted rates are in Aizawl in the north eastern part of India at 24.3 per 100,000 women. ${ }^{1}$ In this study, maximum number of patients, $30(60 \%)$ belonged to FIGO stage IIB. 10 (20\%) patients have stage III B disease, $6(12 \%)$ have stage IVA disease. 4 (8\%) have stage IIA disease and none of the patient in the category of stage IIIA disease. Present findings are similar to Panici et al who found maximum patients in stage IIB followed by stage IIIB and stage IIA. ${ }^{2}$

In present study, $58 \%$ patients were from rural area and $42 \%$ belonged to urban areas. The increased occurrence of cancer cervix in rural areas is because to the fact that $80 \%$ of Indian population resides in rural area. Apart from that, it may be due to presence of number of high risk factors in rural population. They are low socioeconomic status, marriage at an early age, high parity, male sexual behavior, smoking in females and deficiency of micronutrients. Our observation depicts higher incidence of carcinoma cervix in Hindu population as compared to Muslims. This difference in mainly due to lack of circumcision in Hindu males. Wynder et al also suggested that lack of circumcision was an important factor in the etiology of carcinoma cervix. ${ }^{6}$

Present study shows the higher incidence of cervical cancer in lower socioeconomic group than those in higher income groups. Cervical cancer risk is associated with a low socio-economic status (SES), as defined by education or income levels by Parikh et al. ${ }^{7}$ The reasons for the association are not fully understood but are thought to include a higher prevalence of cervical cancer risk factors, such as inadequate cervical cancer screening by Khan et al, high parity by International Collaboration of
Epidemiological Studies of Cervical Cancer, and possibly high-risk sexual behavior by Louie et al among women with a low SES by de Sanjose et al. ${ }^{8-11}$ Risk of carcinoma cervix is inversely related to age at first intercourse. The present study also showed that $60 \%$ of patients got married at an age of 18 years or less. Similar link between cervical neoplasia and early age at first coitus has been reported by Louie et al. ${ }^{10}$

Out of 25 patients receiving chemotherapy, 22 patients did well with complete response in $68.0 \%$ and partial response in $20 \%$. Thus, the overall response rate was $88 \%$. In 18 patients receiving neoadjuvant chemotherapy followed by surgery or radiotherapy, $66.66 \%$ showed CR and $22.22 \%$ showed PR. Two patients $(11.11 \%)$ showed progressive disease while on chemotherapy.

Recently results associated with a combination of initial chemotherapy followed by definitive treatment have been reported and the response rate ranges fom $38-78 \% .{ }^{12-13}$ The reason for relatively high response rate in present study was probably related to maximum number of patients in FIGO stage IIB. Out of seven patients receiving concurrent chemoradiation in this study, 6 patients did well with $71.4 \%$ showing CR and $14.3 \%$ showing partial response and only one patient showed minimal response. Thus, results of concurrent chemoradiation was better as compared to neoadjuvant chemotherapy.

Concurrent use of cisplatin or cisplatin and 5-FU combination have been shown to be a radiosensitizer and have shown additive cytotoxicity in combination with radiotherapy. In control group receiving radiotherapy, $56 \%$ patients showed CR, 36\% showed PR and $8 \%$ showed progressive disease. Neoadjuvant chemotherapy by reducing the tumour bulk and downstaging the disease could make radical surgery possible in a higher percentage of patients previously considered inoperable. Moreover, frequency of lymph node metastasis in 
patients subjected to neoadjuvant chemotherapy was less than excepted for same stage and tumour size. In present study we had been able to perform surgery in two patients with stage IIB disease who were down staged by neoadjuvant chemotherapy.

Authors have found that surgery was not so difficult, and the chemotherapy did not appear to adversely affect the post-operative course. Moreover, in both the patients, no lymph node metastasis was found, and resected margin was free of disease on histopathological examination. However, one of the patient developed recurrence after 10 weeks and received adjuvant pelvic radiotherapy post operatively. This finding in present study suggests the possibility that initial chemotherapy might be able to eliminate the metastasis disease in lymph nodes and possibly micrometastasis. But the problem which came with neoadjuvant chemotherapy is in follow up of patients because the duration of treatment is long, and it requires repeated admissions. In present study we had been able to follow the patients to some extent because we ensured and provided most of them the chemotherapeutic drugs free of cost.

Concurrent chemoradiation showed best treatment outcome, though incidence of effects and toxicity was more as compared to patients receiving neoadjuvant chemotherapy followed by surgery or radiotherapy. Apart from its good response rate, duration of treatment was short with concurrent therapy. It did not require repeated admissions and treatment was completed in a single setting. Thus, problem of losing a patient in intermittent therapy was not there.

Maximum numbers of patients entered in present study were in FIGO stage IIB. Out of 30 patients in stage IIB disease, $70 \%$ showed CR, $16.66 \%$ showed PR whereas $6.66 \%$ showed SD and $6.66 \%$ showed PD while on chemotherapy. Out of 10 patients diagnosed as stage IIIB, $40 \%$ showed CR, $50 \%$ showed PR whereas $10 \%$ showed $\mathrm{SD}$. CR was seen in maximum number of stage IIB patients followed by stage IIA, stage IIIB and markedly less with stage IVA disease. Panici et al in his study of neoadjuvant chemotherapy in locally advanced cancer cervix found higher overall response rate of $88 \%$ in patients with stage IIB disease followed by stage IIA $(78 \%)$ and stage IIIB (74\%). ${ }^{2}$

\section{CONCLUSION}

Chemotherapy is an effective mode of therapy with acceptable side effects and toxicity and hence can be considered as an adjuvant to available modalities of treatment, like surgery or radiotherapy, to improve the overall survival of cancer cervix patients.

Funding: No funding sources

Conflict of interest: None declared
Ethical approval: The study was approved by the Institutional Ethics Committee

\section{REFERENCES}

1. ICO Information Centre on HPV and cancer (Summary Report 2014-08-22). Human Papillomavirus and Related Diseases in India. 2014

2. Panici PB, Scambia G, Baiocchi G, Greggi S, Ragusa G, Gallo A, et al. Neoadjuvant Chemotherapy and radical surgery in locally advanced cervical cancer. Cancer. 1997;67:372-9.

3. Haskell CM. Cancer treatment. 4th ed. Philadelphia: W.B. Saunders; 1995.

4. Buxton EJ, Meanwell CA, Hilton C, Mould JJ, Spooner D, Chetiyawardana A et al. Combination Bleomycin, Ifosfamide, and Cisplatin Chemotherapy in Cervical Cancer, JNCI. 1989,81(5):359-61.

5. Kaushal V, Goel A. Chemotherapy for carcinoma cervix : with what and when? Obstet Gynae. 2001;3:171-4.

6. Wynder El, Cornfield J, Schroff PD, Doraiswami KR. A study of environmental factors in carcinoma of the cervix. Am J Obstet Gynecol. 1954;68(4):1016-52.

7. Parikh S, Brennan P, Boffetta P. Meta-analysis of social inequality and the risk of cervical cancer. Int J Cancer. 2003;105:687-91

8. Khan MJ, Partridge EE, Wang SS, Schiffman M. Socioeconomic status and the risk of cervical intraepithelial neoplasia grade 3 among oncogenic human papillomavirus DNA-positive women with equivocal or mildly abnormal cytology. Cancer 2005;104:61-70.

9. International Collaboration of Epidemiological Studies of Cervical Cancer Cervical carcinoma and reproductive factors: collaborative reanalysis of individual data on 16,563 women with cervical carcinoma and 33,542 women without cervical carcinoma from 25 epidemiological studies. Int $\mathbf{J}$ Cancer. 2006;119:1108-24

10. Louie KS, de Sanjose S, Diaz M, Castellsague X, Herrero R, Meijer CJ, et al (2009) Early age at first sexual intercourse and early pregnancy are risk factors for cervical cancer in developing countries. Br J Cancer. 2009;100:1191-7.

11. de Sanjose S, Bosch FX, Munoz N, Shah K (1997) Social differences in sexual behaviour and cervical cancer. IARC Sci Publ. 1997;138:309-17

12. Friedlander ML, Atkinson K, Coppleson JVM, Elliot P, Green D, Houghton $\mathrm{R}$ et al. The integration of chemotherapy into the management of locally advanced cervical cancer: A pilot study. Gynaecol Oncol. 1984;19:1-7.

13. Desai M, Desai B. Cisplatin MTX chemotherapy before radiotherapy improves survival in cancer cervix. J Obstet Gynecol India. 2001;51:187-9.

Cite this article as: Firdaus $\mathrm{H}$, Ayanat $\mathrm{H}$. Role of chemotherapy in the management of locally advanced carcinoma cervix. Int J Reprod Contracept Obstet Gynecol 2018;7:3275-9. 